\title{
Disputas sobre el sentido de la educación pública: el financiamiento de la "educación pública de gestión privada" en Uruguay
}

\section{Resumen}

Universidad de la República, Montevideo - Uruguay

El artículo indaga sobre las formas de financiamiento de la "educación pública de gestión privada" en Uruguay. Se analiza una modalidad de renuncia fiscal estatal que promueve la realización de donaciones por parte de empresas privadas. Se caracteriza a donantes y donatarios intentando indagar acerca de a existencia de vínculos que apunten a la promoción de procesos de privatización educativa. El trabajo se inscribe en los estudios críticos sobre privatización educativa en desarrollo en América Latina. Opta por una perspectiva teórica centrada en una mirada sobre el rol del Estado en estos procesos. Metodológicamente se trabaja sobre datos secundarios y se sistematiza información presente en sitios webs de instituciones educativas y empresas.

Palabras-claves: Educación Pública. Gestión Privada. Financiamiento Educativo. Estado. Uruguay.

\section{Disputes over de meaning of public education: the financing of privately managed public education in Uruguay}

\begin{abstract}
The article discusses the forms of funding for "public education of private management" in Uruguay. A state tax waiver modality that promotes donations by private companies is discussed. Donors and donatarians are characterized as an attempt to investigate the promotion of educational privatization processes. This work is part of critical studies on educational privatization under development in Latin America. It opts for a theoretical perspective aimed at looking at the role of the State in these processes. Methodologically, secondary data are used and information is systematized from websites of educational institutions and companies.
\end{abstract}

Keywords: Public Education. Private Management. Educational Funding. State. Uruguay. 
Disputas sobre el sentido de la educación pública

\section{Presentación}

El presente texto forma parte de un proyecto de investigación orientado a analizar la problemática de la privatización educativa en Uruguay. En este artículo se toma como objeto de indagación el financiamiento de la "educación pública de gestión privada" a través de la modalidad definida como "Donaciones Especiales". Esta modalidad habilita a empresas privadas a realizar donaciones a instituciones educativas, descontando hasta el $81,25 \%$ de lo donado a través de renuncias fiscales del Estado uruguayo. A su vez, se indaga en torno a las instituciones que reciben las donaciones y a las empresas que las realizan, mostrando la existencia de una conjunción que apunta a promover procesos de privatización educativa.

Se parte de la base de la consideración de trabajos de investigación que han analizado la temática de la privatización educativa a nivel global (BALL; YOUDELL, 2008; BALL, 2011; 2014; BELLEI; ORELLANA, 2014; VERGER et al., 2016), latinoamericano (PERONI, 2013; VERGER et al., 2017; PERONI et al., 2018; CAETANO, 2018) y uruguayo (BORDOLI et al., 2017; MANCEBO, 2018; MOSCHETTI et al., 2019; MARTINIS, 2019; BORDOLI; CONDE, 2020).

Se parte de una concepción de privatización como expresión de lógicas globales que tienden a desmontar la responsabilidad de los estados nacionales en el aseguramiento del derecho a la educación y que sostienen soluciones de mercado para los problemas de la educación (BALL, 2014).

Desde el punto de vista metodológico el trabajo se ha organizado a través de la recopilación de informes producidos a nivel gubernamental en Uruguay acerca de las formas de organización, los procedimientos y los montos donados en el marco de la modalidad de Donaciones Especiales. También se ha recurrido a información ofrecida en páginas web tanto de empresas donantes como de instituciones educativas beneficiarias.

En cuanto a la estructura del texto, se organiza en cinco apartados. El primero explicita las formas de financiamiento de la educación privada en Uruguay. El segundo da cuenta de la evolución del financiamiento por Donaciones Especiales en el período 2010 - 2019. El tercero presenta un perfil general de los donantes. El cuarto refiere a las disputas sobre el sentido de la educación pública a través del direccionamiento del financiamiento de las Donaciones Especiales. Finalmente, el quinto presenta algunas conclusiones del trabajo de investigación.

\section{Las formas de financiamiento público de la educación privada en Uruguay}

En Uruguay no existen formas de subsidio directo por parte del Estado a las instituciones educativas privadas del estilo de las que se han implementado en otros países de América Latina (DOBERTI, 2001; CIPPEC, 2007; LEHER, 2009; IBARRA ROSALES, 2017). No obstante, sí existe una larga tradición de exoneraciones impositivas a estas instituciones, las cuales se presentan por primera vez en la Constitución de la República del año 1942.

A partir del año 2007 la legislación nacional presenta una nueva forma de subsidio. Esta tiene que ver con la habilitación de exoneraciones impositivas a empresas que realicen donaciones a instituciones vinculadas a temáticas de infancia, educación y salud. Este tipo de 
subvenciones va en la línea del financiamiento privado - público al que hacen referencia Moschetti, Fontdevila y Verger (2017) en su trabajo sobre modelos de privatización educativa

A continuación describiremos ambas modalidades como introducción al debate acerca de las formas de promoción del financiamiento público de la educación privada en Uruguay.

\section{Las exoneraciones impositivas}

A partir de la Constitución de la República del año 1942, la enseñanza privada goza de un conjunto de exoneraciones impositivas, las cuales son concebidas como un subsidio estatal que reconoce la labor que realizan. Estas exoneraciones se establecieron como una forma de zanjar las disputas que entre sectores afines a la Iglesia Católica y sectores defensores del Estado laico se habían producido a partir de la separación Estado - Iglesia sancionada en la Constitución de 1917.

Esta normativa se ha mantenido a través de diversas reformas constitucionales. La Constitución actualmente vigente", establece en su artículo 69 que "Las instituciones de enseñanza privada y las culturales de la misma naturaleza estarán exoneradas de impuestos nacionales y municipales, como subvención por sus servicios". Esta norma difícilmente podría ser comprendida si no se la coloca en el marco del artículo que la precede en el texto constitucional. El artículo 68 establece: "Todo padre o tutor tiene derecho a elegir, para la enseñanza de sus hijos o pupilos, los maestros o instituciones que desee".

Complementariamente, la Ley 16226 (1991), que estableció el beneficio de las exoneraciones fiscales comprende "a las Instituciones privadas que tienen como finalidad única o predominante la enseñanza privada o la práctica o difusión de la cultura". Como han sistematizado Bordoli et al. (2017), las exoneraciones abarcan todos los impuestos de tipo nacional o departamental, lo cual implica:

a) Aportes patronales a la seguridad social jubilatorios y al Fondo Nacional de Salud (FONASA) [...], b) Impuesto de primaria: impuesto anual que grava a las propiedades inmuebles urbanas, suburbanas y rurales. La recaudación del impuesto se destina a financiar los créditos presupuestales de gastos e inversiones del Consejo de Educación Primaria, c) Impuesto a las rentas de las actividades económicas (IRAE): impuesto anual sobre las rentas de fuente uruguaya de actividades económicas de cualquier naturaleza, Impuesto al patrimonio: impuesto anual que recae sobre el patrimonio de las personas físicas, los núcleos familiares y las sucesiones indivisas, siempre que su patrimonio fiscal exceda del mínimo no imponible respectivo, d) Adquisición de automotores, que incluye los siguientes tributos: la tasa global arancelaria de la Dirección Nacional de Aduanas, IMESI (impuesto específico interno) y de IVA (impuesto al valor agregado), e) Importación de material educativo, que incluye los siguientes tributos: la tasa global arancelaria de la Dirección Nacional de Aduanas y de IVA, f) Contribución inmobiliaria: es un impuesto municipal que se cobra en relación al valor real del Padrón, g) Tributos municipales como la patente de rodados, tasas de alumbrado, saneamiento, etc. (BORDOLI et al., 2017, p. 25-26).

La participación del sector privado en la educación uruguaya ha sido definido en diversas investigaciones como moderado o bajo en comparación con los niveles

1 Se trata de la Constitución de 1967 a la cual se han introducido ajuste a través de las reformas de los años 1990, 1995 y 1997. 
latinoamericanos (VERGER et al., 2017). Siempre se ha ubicado por debajo del $20 \%$ en la globalidad de la matrícula de la enseñanza básica.

Para el año 2018, según datos del Ministerio de Educación y Cultura de Uruguay (URUGUAY, 2018), la participación de la educación privada en el total de la matrícula es la que puede apreciarse en el siguiente cuadro.

Tabla 1 - Participación de la educación privada en el total de la matrícula en los niveles primario y medio en Uruguay. Año 2018

\begin{tabular}{|l|r|r|r|}
\hline & \multicolumn{2}{|l|}{ Total matrícula } & \multicolumn{2}{l|}{ Matrícula privada } & \multicolumn{2}{l|}{$\begin{array}{l}\text { \% de participación } \\
\text { enseñanza privada }\end{array}$} \\
\hline Primaria Común & 294.291 & 51.289 & 17,4 \\
\hline Media Básica y Superior & 269.457 & 41.221 & 15,3 \\
\hline Total & 563.748 & 92.510 & 16,4 \\
\hline
\end{tabular}

Fuente: Elaboración propia en base a datos de MEC (2018).

Debido a la heterogeneidad de la oferta y a la inexistencia de datos agregados, resulta sumamente complejo establecer con claridad los montos de recursos a los que el Estado renuncia como consecuencia de las exoneraciones otorgadas a la enseñanza privada.

Un estudio realizado por el Instituto Nacional de Evaluación Educativa (INEED, 2017) intentó avanzar sobre esta temática, constituyendo la aproximación más destacada a esta realidad. A través de una compleja metodología que cruza información tanto de las instituciones de enseñanza privada como de organismos estatales encargados de la recaudación impositiva, se establece que el conjunto de la renuncia fiscal realizada por el Estado uruguayo se ubicaba en el año 2015 en el 9\% del total de los recursos que destina a financiamiento educativo. Esta renuncia fiscal, se compone en un $97,1 \%$ de exoneraciones de cargas impositivas y aportes a la seguridad social de instituciones privadas. El restante $2.9 \%$ corresponde a exoneraciones fiscales a empresas por donaciones realizadas en el marco de la Ley 18.834, a las que nos referiremos más adelante (INEED, 2017).

De la información aportada por el estudio mencionado puede establecerse que si bien el estado uruguayo no dispone transferencias monetarias directas hacia la educación privada, las exoneraciones que realiza constituyen una forma de subsidio indirecto relevante. De hecho se destina como subsidio indirecto el $9 \%$ de los recursos destinados a la educación a una modalidad educativa que atiende al $16,4 \%$ de la matrícula en educación primaria, media básica y media superior del país.

\section{Las exoneraciones fiscales a empresas donantes}

Uno de los componentes que alimentó la expansión de los postulados privatizadores en Uruguay fue el uso que un sector del empresariado, aliado con otros actores, hizo del instrumento de las "Donaciones Especiales" (BORDOLI et al., 2017; MOSCHETTI et al., 2019). Este instrumento se introdujo en la legislación en la reforma tributaria de 2007 (Ley 18083), la cual habilita que empresas privadas realicen donaciones a instituciones públicas o privadas que trabajan en temáticas de educación, infancia carenciada y salud, pudiendo 
descontar impositivamente el $81,25 \%$ de lo donado. El marco ha sido luego perfeccionado a través de diversas normativas.

La ley 18834 (año 2012) establece que las instituciones beneficiarias deberán presentar un proyecto para poder participar de esta modalidad, a la vez que se obligan a presentar un informe luego de finalizar la ejecución. También establece que el Poder Ejecutivo podrá establecer montos máximos a ser donados por las empresas y también topes a los recursos que pueden ser recibidos.

La ley 19149 (año 2014) en su artículo 358 establece precisiones en cuanto al tipo de instituciones que podrán recibir donaciones. La ley 19438 (año 2018) en su artículo 188 establece nuevos procedimientos administrativos. La ley 19670 (año 2018), en su artículo 351, incorpora nuevas instituciones de diversos ámbitos entre las que pueden recibir donaciones.

Si bien, como ya se ha señalado, esta modalidad de financiamiento educativo involucra montos que son marginales dentro del conjunto de los recursos destinados a la educación en Uruguay, su relevancia ha sido fundamental para permitir el desarrollo de experiencias educativas organizadas por actores privados que se han propuesto poner en discusión la hegemonía de la educación pública. Particularmente, estas experiencias se han posicionado como alternativas frente a lo que se define como el fracaso de la educación en contextos de pobreza en nuestro país (MARTINIS, 2019).

\section{La evolución del destino de las Donaciones Especiales en el período 2010 - 2019}

Un primer elemento que interesa mencionar con respecto al instrumento de las Donaciones Especiales, es que las mismas pueden ser realizadas tanto a instituciones públicas como a privadas. Es posible suponer que al promover este instrumento en el 2007, los legisladores del partido político de centro izquierda Frente Amplio, que gobernó el país entre los años 2005 y 2019, tuvieran en mente la posibilidad que sirviera para lograr transferencia de recursos al sector público.

Durante los dos primeros años de ejecución de esta modalidad de donaciones, efectivamente el monto mayoritario se destinó a experiencias educativas públicas. No obstante, a partir del tercer año, el 2012, esta tendencia se alteró radicalmente. En el siguiente cuadro mostramos la participación de la educación pública en el conjunto de las donaciones destinadas a la educación primaria, media básica y media superior.

Tabla 2 - Porcentaje de Donaciones Especiales recibidas por la educación pública 2010 - 2019

\begin{tabular}{|l|r|r|r|r|r|r|r|r|r|r|}
\hline Año & 2010 & 2011 & 2012 & 2013 & 2014 & 2105 & 2016 & 2017 & 2018 & 2019 \\
\hline $\begin{array}{l}\text { Total donado a } \\
\text { instituciones } \\
\text { públicas y } \\
\text { privadas (en } \\
\text { millones de } \\
\text { pesos) }\end{array}$ & 31,8 & 45,4 & 29,0 & 53,0 & 108,8 & 118,6 & 115,8 & 130,6 & 155,9 & 156,2 \\
\hline $\begin{array}{l}\text { Porcentaje } \\
\text { recibido por } \\
\text { educación } \\
\text { pública }\end{array}$ & 98,4 & 59,5 & 16,6 & 8,9 & 7,8 & 11,0 & 7,9 & 5,8 & 4,8 & 3,4 \\
\hline
\end{tabular}

Fuente: Elaboración propia en base a datos de MEF (2019) y MEF (2020). 
Disputas sobre el sentido de la educación pública

Una primera conclusión de suma relevancia para nuestro trabajo que se puede desprender de la tabla presentada, es que a medida que el instrumento fue tomando mayor desarrollo, las donaciones dirigidas a la educación pública tendieron a decrecer rápidamente en relación con el total de lo donado. Como puede apreciarse, salvo un par de excepciones que no alteran la tendencia, el monto total de lo donado fue incrementándose año tras año. De hecho, si tomamos los dos extremos de la serie presentada, puede apreciarse que el monto donado en 2019 prácticamente quintuplica al del año 2010.

Una forma de valorar el incremento real de los montos que figuran en la tabla es establecer que, según datos tomados del Banco Central del Uruguay², la variación del valor del dólar interbancario entre los últimos días de junio de 2010 (cotización: 21,127) y de 2019 (cotización: 35,182) fue de $66,5 \%$ al alza. Otro indicador relevante surge de apreciar que según datos del Instituto Nacional de Estadística ${ }^{3}$ la variación en el Índice de Precios al Consumo entre junio de 2010 (índice 96.41) y junio de 2019 (índice 196,44) fue del 103, 8\%. Tomamos en ambos casos el mes de junio para ubicarnos en un punto intermedio del año.

La distancia entre ambas cifras y el crecimiento efectivamente apreciado en los montos de las donaciones, muestra que el incremento fue significativo. Este incremento prácticamente cuadruplicó el del Índice de Precios al Consumo y septuplicó el del valor del dólar.

Un segundo elemento a destacar en el análisis es el incremento sustantivo de las donaciones recibidas por instituciones privadas. A los efectos de calibrar adecuadamente este fenómeno resulta imprescindible presentar la información del total de las instituciones de enseñanza primaria, media básica y media superior que recibieron aportes en el período. Ello puede apreciarse en la siguiente tabla.

2 Datos en: <https://www.bcu.gub.uy/Estadisticas-e-Indicadores/Paginas/Cotizaciones.aspx>.

3 Datos en: <http://www.ine.gub.uy/indicadores?indicadorCategoryld=11421>. 
Tabla 3 - Monto recibido por institución expresado en millones de pesos uruguayos. Período $2010-2019$

\begin{tabular}{|c|c|c|c|c|c|c|c|c|c|c|c|}
\hline Institución & 2010 & 2011 & 2012 & 2013 & 2014 & 2015 & 2016 & 2017 & 2018 & 2019 & TOTAL \\
\hline $\begin{array}{l}\text { Fundación } \\
\text { Impulso (Liceo } \\
\text { Impulso) }\end{array}$ & & 12,1 & 16,6 & 35,5 & 66,2 & 64,0 & 58,0 & 68,6 & 73,1 & 79,0 & 473,1 \\
\hline ANEP & 31,3 & 27,0 & 4,8 & 4,7 & 8,5 & 13,1 & 9,1 & 7,5 & 7,2 & 5,3 & 118,5 \\
\hline $\begin{array}{l}\text { Liceo Jubilar } \\
\text { Juan Pablo II }\end{array}$ & 0,5 & 6,3 & 7,6 & 11,8 & 13,4 & 15,0 & 16,2 & 12,0 & 18,9 & 15,5 & 117,2 \\
\hline Liceo Providencia & & & & & 11,3 & 11,0 & 12,0 & 13,0 & 15,0 & 17,8 & 80,1 \\
\hline Liceo Francisco & & & & & 9,4 & 9,1 & 10,0 & 10,8 & 11,5 & 12,4 & 63,2 \\
\hline $\begin{array}{l}\text { Bachillerato } \\
\text { Tecnológico } \\
\text { Ánima }\end{array}$ & & & & & & 3,5 & 8,0 & 8,6 & 9,2 & 9,9 & 39,2 \\
\hline $\begin{array}{l}\text { Fundación } \\
\text { Sophía }\end{array}$ & & & & & & & & 6,2 & 12,5 & 8,7 & 27,4 \\
\hline $\begin{array}{l}\text { Colegio Sagrado } \\
\text { Corazón }\end{array}$ & & & & & & 2,9 & 2,5 & 3,1 & 2,7 & 3,0 & 14,2 \\
\hline Los Rosales & & & & & & & & & 5,0 & & 5 \\
\hline $\begin{array}{l}\text { Colegio Obra } \\
\text { Banneux }\end{array}$ & & & & & & & & & & 2,6 & 2,6 \\
\hline $\begin{array}{l}\text { Liceo San José } \\
\text { de Tala }\end{array}$ & & & & & & & & 0,8 & 0,8 & 0,9 & 2,5 \\
\hline Colegio Belén & & & & & & & & & & 1,1 & 1,1 \\
\hline $\begin{array}{l}\text { Colegio María } \\
\text { Auxiliadora }\end{array}$ & & & & 1,0 & & & & & & & 1,0 \\
\hline TOTAL & 31,8 & 45,4 & 29,0 & 53,0 & 108,8 & 118,6 & 115,8 & 130,6 & 155,9 & 156,2 & 945,1 \\
\hline
\end{tabular}

Fuente: Elaboración propia en base a datos de MEF (2019) y MEF (2020).

En la tabla precedente se sistematiza el total de las donaciones recibidas por todas las instituciones beneficiarias en el período 2010 -2019. Salvo la información referida a la Administración Nacional de Educación Pública ${ }^{4}$ (ANEP), la cual comprende donaciones recibidas centralmente por la ANEP que luego son volcadas a diversos proyectos específicos, todas las demás referencias son de instituciones privadas. Entre estas, podemos encontrar tres tipos de organizaciones: a) la Fundación Sophia pertenece a la Iglesia Católica y tiene entre sus cometidos el apoyo pedagógico y organizacional a instituciones educativas de esa denominación religiosa ubicadas en zonas de vulnerabilidad social; b) los colegios Sagrado Corazón, Los Rosales, Obra Banneux, San José de Tala, Belén y María Auxiliadora son instituciones tradicionales pertenecientes a la Iglesia Católica que se financian en parte por

\footnotetext{
4 La Administración Nacional de la Educación Pública es el ente autónomo que en Uruguay tiene a su cargo la educación inicial, primaria, media básica, media superior y la formación de docentes. Sus funciones corresponden a las que en otros países recaen sobre los Ministerios de Educación.
} 
recursos de las familias y en parte por donaciones; finalmente, c) los Liceos Impulso (desarrollado por la Fundación Impulso), Jubilar, Providencia, Francisco y el Bachillerato Tecnológico Ánima, son un nuevo tipo de instituciones educativas autodefinidas como "públicas de gestión privada" que se financian exclusivamente por donaciones, no cobrando matrículas a sus estudiantes. La primera es de carácter laico y las restantes pertenecen a la Iglesia Católica. Es de señalar que el carácter de públicas que se asignan estas instituciones remite exclusivamente a su carácter gratuito, lo cual implica una fuerte banalización del sentido del término. Son las instituciones pertenecientes al tercer grupo las que reciben mayores montos de donaciones. Estas instituciones fueron parte de una estrategia desarrollada por un mix de sectores liberales y conservadores destinada a disputar la centralidad de la educación pública en la sociedad uruguaya. Particularmente su estrategia se basó en mostrar, a partir de su inserción en zonas de alta vulnerabilidad, que sus proyectos educativos eran capaces de lograr resultados educativos netamente superiores a los obtenidos por la educación pública (MARTINIS, 2019).En la tabla que se presenta a continuación sistematizamos la información en cuanto a los porcentajes del total de las donaciones realizadas en el período 2010 - 2019 recibidas por cada institución. Como es posible apreciar, un porcentaje cercano al $85 \%$ es percibido por cuatro donatarios. Se destaca ampliamente el porcentaje prácticamente equivalente a la mitad de las donaciones que es percibido por el Liceo Impulso.

Tabla 4 - Total de donaciones realizadas en el período 2010 - 2019 y porcentaje correspondiente a cada institución

\begin{tabular}{|l|r|r|r|}
\hline Institución & \multicolumn{1}{|l|}{$\begin{array}{l}\text { Total } \\
\text { donaciones } \\
\mathbf{2 0 1 0 - 2 0 1 9}\end{array}$} & $\begin{array}{l}\text { Porcentaje del } \\
\text { total en el período }\end{array}$ & $\begin{array}{l}\text { Porcentaje } \\
\text { acumulado }\end{array}$ \\
\hline Fundación Impulso & 473,1 & 50,06 & 50.06 \\
\hline ANEP & 118,5 & 12,54 & 62,6 \\
\hline Liceo Jubilar Juan Pablo II & 117,2 & 8,48 & 75,0 \\
\hline Liceo Providencia & 80,1 & 6,68 & 83,48 \\
\hline Liceo Francisco & 63,2 & 4,14 & 90.16 \\
\hline Bachillerato Tecnológico Ánima & 39,2 & 2,90 & 94,3 \\
\hline Fundación Sophía & 27,4 & 1,5 & 97,2 \\
\hline Colegio Sagrado Corazón & 14,2 & 0,53 & 98,7 \\
\hline Los Rosales & 5 & 0,28 & 99,23 \\
\hline Colegio Obra Banneux & 2,6 & 0,26 & 99,51 \\
\hline Liceo San José de Tala & 2,5 & 0,12 & 99,77 \\
\hline Colegio Belén & 1,1 & 0,11 & 99,89 \\
\hline Colegio María Auxiliadora & 945,1 & 100 & 100 \\
\hline TOTAL & 1,0 & & \\
\hline
\end{tabular}

Fuente: Elaboración propia en base a datos de MEF (2019) y MEF (2020).

Es de hacer notar que más allá del porcentaje de donaciones que percibe la ANEP, las cuales la ubican en el segundo lugar en cuanto a monto recibido, la sumatoria de tres 
instituciones privadas (Liceos Impulso, Jubilar y Providencia) permite explicar el 70,94 \% del total de lo donado. Si excluimos lo recibido por ANEP en el cálculo total, el porcentaje recibido por estas tres instituciones llega a explicar el $71,89 \%$ de lo donado. Teniendo este dato en cuenta, y a modo de avance de estudios que deberán indagar sobre el conjunto de los donatarios, nos parece interesante detenernos en estas tres instituciones a los efectos de presentar sumariamente sus propuestas institucionales y dar cuenta, en el próximo apartado, del perfil de los donantes que han participado en esta modalidad de financiamiento educativo. Interesa observar a estos donatarios en detalle ya que ellos han sido quienes han llevado adelante una estrategia que ha permitido redirecionar recursos de erario público hacia el financiamiento de instituciones educativas privadas.

El Liceo Impulso surge a partir de la conformación de la Fundación del mismo nombre en el año 2010. Entre los socios fundadores figuraban dos personalidades estrechamente vinculadas a la derecha política uruguaya: Ernesto Talvi y Pablo da Silveira. El primero de ellos, economista, fue responsable del Centro de Estudios de Estudios de la Realidad Económica y Social (CERES), uno de los principales think tank neoliberales del Uruguay, asociado a Atlas Network en América Latina ${ }^{5}$. Posteriormente, fue candidato a la presidencia de la República por uno de los dos partidos históricos y de raigambre liberal del país, el Partido Colorado. Luego de la primera vuelta electoral realizada en octubre de 2019 apoyó en el ballotage de noviembre del mismo año al candidato que sería electo Presidente de la República: Luis Lacalle Pou. El nuevo presidente, que asumió el 1 de marzo de 2020 ganó las elecciones apoyado por una coalición de cinco partidos que abarca desde posiciones de centro político hasta la ultraderecha militar. En este gobierno, Talvi ocupó la posición de Ministro de Relaciones Exteriores entre el 1 de marzo y el 1 de julio de 2020.

Por otra parte, Pablo da Silviera es un destacado intelectual uruguayo vinculado a la Universidad Católica. En sus trabajos académicos ha abogado por las soluciones de mercado a las problemáticas educativas (DA SILVEIRA, 1995). Fue uno de los principales asesores del actual presidente Lacalle Pou. Actualmente es Ministro de Educación y Cultura de su gobierno.

Otros de los integrantes de la comisión directiva de la Fundación Impulso son: Nicolás Herrera, uno de los principales donantes de Impulso como se puede apreciar en la Tabla 5; Elvio Strauch, vinculado al Banco Santander, otro donante de Impulso; y Horacio Hughes, vinculado a la financiera OCA, otro de los grandes donantes de Impulso.

Realizamos esta breve descripción de las autoridades de la Fundación Impulso para intentar dar cuenta como en su seno se reúnen una serie de figuras relevantes de los ámbitos políticos, académicos y económicos del país a los efectos de generar una propuesta que dispute espacios a la educación pública.

El Liceo Impulso se ubica en la zona del barrio Casavalle de Montevideo, zona marcada por uno de los niveles de pobreza más altos del país. En ese contexto, según se plantea en su sitio web, el Liceo se define como:

[...] liceo gratuito, laico y de gestión privada, que desarrolla al máximo la potencialidad de sus alumnos con el máximo de exigencia, orientado a generar oportunidades para la

5 La información sobre CERES ha sido tonada de su web institucional: <https://ceres-uy.org>. 
Disputas sobre el sentido de la educación pública

búsqueda del desarrollo personal y la excelencia académica, basado en los valores de auto-respeto, ejercicio responsable de la libertad, disciplina y cultura del esfuerzo ${ }^{6}$.

Por otra parte, en la misma página web se puede leer que la institución tiene como objetivos:

Eliminar la brecha de aprendizajes en los adolescentes de la Cuenca del Casavalle. Para ello desarrollamos un liceo con una cultura muy fuerte del aprendizaje, basados en la excelencia, logrando un aprendizaje integral que permita ampliar las capacidades y horizonte de oportunidades reales y significativas de los alumnos ${ }^{7}$.

Si bien excede nuestros objetivos adentrarnos en una revisión detallada de cada una de las instituciones que reciben donaciones, entendemos que estas referencias son suficientes para comprender el perfil institucional y su apuesta como un espacio gratuito, de gestión privada y que apunta a la excelencia en un contexto de pobreza urbana. Desde estas posiciones, la institución disputa sentidos con la educación pública, ofreciéndose como alternativa educativa en un contexto en el cual el sistema educativo uruguayo se encuentra con importantes dificultades para el logro de buenos resultados educativos.

Los liceos Jubilar y Providencia presentan un perfil similar al del Impulso, se ubican en zona de pobreza urbana aunque no se presentan como laicos sino como parte de la Iglesia Católica. Se trata de instituciones que se distinguen por su carácter gratuito y la gestión privada. También en sus presentaciones en la web ${ }^{8}$ se destaca su carácter de instituciones que buscan construir alternativas para jóvenes pobres a través de la educación. Un elemento destacado de los sitios web de ambas instituciones, que no se aprecia en el de Impulso, es su presentación explícita de las empresas y fundaciones que los apoyan económicamente. En el caso del Liceo Jubilar se hace referencia a 39 organizaciones de este tipo, y en el caso de Providencia las referencias llegan a 72 organizaciones. En esos listados se encuentra presentes muchas de las empresas que aparecen incluidas en la tabla 5, así como otras tantas que realizan donaciones por fuera de la modalidad de Donaciones Especiales. Si bien no ha sido posible todavía sistematizar los aportes de estos otros donantes, acceder a esa información sería de suma relevancia a los efectos de poder dar cuenta más cabalmente de la incidencia de los recursos que destinan empresas privadas al financiamiento de esa modalidad educativa en el país.

En su conjunto estas tres instituciones, al igual que otras que participan de esta modalidad de donaciones especiales, han sido presentadas como instituciones ejemplares en lo que tiene que ver con el logro de buenos resultados educativos en contextos de pobreza. A partir de ellas se desarrolló en la sociedad uruguaya una reivindicación de las bondades de la llamada "educación pública de gestión privada" (DUFRECHOU et al., 2019).

En el siguiente apartado nos detendremos en un análisis de los grandes donantes que hacen posible el financiamiento de las experiencias de "educación pública de gestión privada". Es importante dejar en claro que estas donaciones parecen no solamente realizarse en función de fines altruistas, sino que también tienen que ver con el fomento de modalidades educativas que disputan sentidos a la educación pública, transmitiendo un mensaje con

6 Referencia tomada de: <http://www.liceoimpulso.edu.uy/reservations>.

7 Idem.

8 Web Jubilar: <https://www.liceojubilar.edu.uy>. Web Providencia: <http://www.providencia.org.uy>. 
Disputas sobre el sentido de la educación pública

respecto a la conveniencia de apostar a soluciones filantrópicas guiadas por una lógica privada a los problemas de la educación uruguaya (MANCEBO, 2018).

\section{Perfil general de los grandes donantes}

A los efectos de avanzar en la caracterización de los donantes, optamos por concentrarnos en los aportes recibidos por las tres instituciones privadas que más donaciones recibieron en el período 2017 - 2019. Este recorte se justifica en que son estos los años en que se realizaron mayores donaciones. El acumulado de los tres años explica casi la mitad $(46,84 \%)$ del total donado en el período 2010 - 2019.

El objetivo de nuestro trabajo en este período se dirigió a identificar a lo que llamamos "grandes donantes", esto es, aquellos que aportaron mayores cantidades de dinero en el período. Para ello, en primera instancia nos detuvimos en un análisis de los donantes de la Fundación Impulso, por el peso relativo de esta Fundación en el conjunto de las donaciones.

En el caso de esta Fundación realizamos una revisión del conjunto de las donaciones percibidas en el período 2010 - 2019, ubicando en el mismo todos aquellos donantes que al menos hubieran donado un millón de pesos en alguno de los años comprendidos en el período. Esto nos permitió un primer acercamiento a la caracterización de grandes donantes. De este relevamiento surgió que 33 donantes cumplían con este requisito.

A continuación, analizamos las donaciones recibidas por los liceos Jubilar y Providencia entre 2017 y 2019, prestando atención a dos elementos: a) relevar aquellos donantes que hubieran aportado al menos 1.000 .000 de pesos en el período, y b) relevar donantes que figuraran también en la nómina del Liceo Impulso, aunque hubieran donado menos de 1.000.000 de pesos a estas dos instituciones. Con esto, pretendimos tanto identificar nuevos donantes significativos como consolidar información referida a donantes ya relevados por sus aportes al Liceo Impulso.

Con este procedimiento llegamos a un total de 39 donantes. Un elemento relevante que surgió en primera instancia de este cruzamiento de información, es que estos donantes tendían a realizar aportes a más de una de las instituciones. De hecho, encontramos que 24 donantes realizaban donaciones a las tres instituciones, 5 a dos instituciones y 10 donaron a una sola de las tres instituciones. Esto nos permite avizorar que la existencia de una cierta coordinación en cuanto al destino de as donaciones realizadas.

A cuenta de futuros trabajos que deberán continuar profundizando en el conjunto de la información obtenida en el marco de la investigación en la que se inscribe este artículo, optamos por detener nuestra mirada en las 15 empresas que realizan donaciones más voluminosas en el período analizado. La información sobre los montos donados por cada empresa por año pueden apreciarse en la siguiente tabla. 
Disputas sobre el sentido de la educación pública

Tabla 5 - Grandes donantes y montos donados en el período 2017 - 2019 en millones de pesos uruguayos

\begin{tabular}{|c|c|c|c|c|}
\hline & 2017 & 2018 & 2019 & TOTAL \\
\hline $\begin{array}{l}\text { Tenaris Global Services } \\
\text { S.A. }\end{array}$ & 6585794 & 12639525 & 14914000 & 34139319 \\
\hline Nicolás Herrera y Otros & 8744000 & 4002000 & 5540000 & 18286000 \\
\hline Enalur S.A. & 4500000 & 4950000 & 5100000 & 14550000 \\
\hline OCA S.A. & 4130615 & 3909200 & 3368000 & 11407815 \\
\hline $\begin{array}{l}\text { Supermercados Disco del } \\
\text { Uruguay S.A. }\end{array}$ & 3050000 & 3300000 & 3630000 & 9980000 \\
\hline TCU S.A. & 3000000 & 3000000 & 3220000 & 9220000 \\
\hline Pamer S.A. & 2190000 & 3285000 & 3710080 & 9185080 \\
\hline Compañía Cibeles S.A. & 3050000 & 2500000 & 3500000 & 9050000 \\
\hline Banco Itaú Uruguay S.A. & 2756063 & 2886000 & 2886000 & 8528063 \\
\hline FNC S.A. & 2853840 & 3281916 & 2000000 & 8135756 \\
\hline $\begin{array}{l}\text { Arcos Dorados Uruguay } \\
\text { S.A. (Mc Donalds) }\end{array}$ & & 3800000 & 4330185 & 8130185 \\
\hline Frig. Tacuarembó & 2417000 & 2610000 & 2610000 & 7637000 \\
\hline Vivion S.A. & 2097000 & 3530000 & 2000000 & 7627000 \\
\hline Jelsi S.A. & 2356782 & 4470000 & 680000 & 7506782 \\
\hline Citibank S.A. Uruguay & 2159100 & 2355000 & 2737500 & 7251600 \\
\hline
\end{tabular}

Fuente: elaboración propia en base a MEF (2018) y MEF (2019).

Las 15 entidades incluidas en la tabla precedente constituyen lo que hemos denominado "grandes donantes". Es de destacar que entre las mismas figuran catorce empresas y una persona que dona a título personal y en representación de otros. Este último, Nicolás Herrera, es uno de los integrantes del estudio jurídico, contable e impositivo más importante del país, asesor de grandes empresas a nivel local e internacional. Se trata del estudio Guyer Regules. A su vez, como ya hemos visto, integra la Comisión Directiva de la Fundación Impulso. Se trata de un ejemplo de conjunción de perfil empresarial con activismo social desde perspectivas liberales, ya que también integra la selecta Sociedad de Mont Pelerin ${ }^{9}$.Del resto de las empresas, solamente tres corresponden a emprendimientos compuestos fundamentalmente por capitales nacionales. Se trata de la papelera PAMER, dedicada al rubro de papel para corrugar y envases de cartón corrugado; la compañía CIBELES, dedicada al mercado de la salud humana, animal y vegetal, y, JELSI, dedicada al rubro de la construcción.

9 Una síntesis del currículum vitae de Herrera puede apreciarse en: <https://www.guyer.com.uy/es/professionals/herreranicolas/>. 
Las 11 empresas restantes correspondan todas a empresas transnacionales con sede en Uruguay. El principal donante, Tenaris, opera en el mercado global del acero. Luego se presentan empresas dedicadas a las telecomunicaciones (ENALUR), al ámbito financiero (OCA, ITAÚ y CITIBANK), al supermercadismo (DISCO), a la gestión de una terminal aeroportuaria de cargas (TCU), a bebidas y alimentación (FNC, Mc Donalds y FRIGORÍFICO TACUAREMBÓ), y a la demótica / inmótica (VIVION).

La mirada en conjunto de estos grandes donantes permite apreciar como un grupo de empresas, con fuertes vínculos con el capital trasnacional, ha incidido en el direccionamiento de recursos en el marco del instrumento de las Donaciones Especiales. A modo de hipótesis es posible plantear la posibilidad de la existencia de algún nivel de coordinación entre estas empresas a los efectos de direcciones sus aportes. Lo que sí es claramente visible es que en conjunto son protagonistas de un proceso que tiende a relegar a la educación pública en la recepción de recursos a la vez que hace posible la concentración de los mismos en un número reducido de instituciones definidas como de "educación pública de gestión privada".

\section{Las disputas sobre el sentido de la educación pública a través del direccionamiento del financiamiento de las Donaciones Especiales}

A lo largo de las líneas desarrolladas en los apartados precedentes hemos pretendido mostrar los instrumentos fundamentales a través de los cuales se promueve, a través de renuncias fiscales, el desarrollo de la educación privada en Uruguay. Apreciamos, según datos del Instituto Nacional de Evaluación Educativa (INEED, 2017), que el 9\% de los recursos destinados a financiamiento educativo por parte del Estado uruguayo provienen de esta fuente.

Uno de estos instrumentos se origina en la primera mitad del siglo $\mathrm{XX}$ y tiene que ver con la existencias de exoneraciones impositivas que se aplican a las instituciones educativas privadas. Como también vimos, el $97,1 \%$ de la renuncia fiscal que realiza el Estado uruguayo se explica por esta vía.

El otro instrumento, de origen mucho más reciente y que involucra un monto significativamente menor de recursos es el generado bajo la figura de las "Donaciones Especiales". Básicamente se trata de exoneraciones fiscales que se realizan a empresas privadas en reconocimiento a los aportes que estas brindan a instituciones educativas que operan en contexto de pobreza. Según los datos de INEED (2017), esta modalidad explica el $2,9 \%$ de la renuncia fiscal realizada en el año 2015. Por tanto, bajo este procedimiento se gestiona el $0,26 \%$ de los recursos que el Estado uruguayo destina a financiar la educación.

Si bien el porcentaje puede parecer ínfimo con respecto al total del financiamiento que provee el Estado, resulta importante realizar dos consideraciones al respecto: a) si excluimos las donaciones recibidas por la educación pública podemos apreciar que en el período 2010 - 2019 el 87,46 \% de las donaciones son recibidas por un reducido número de doce instituciones privadas; b) de estas instituciones, una de ellas, la Fundación Impulso, acapara la mitad del monto total donado; y otros dos, los liceos Jubilar y Providencia, reciben un monto levemente superior a la quinta parte de las donaciones. De este modo, es posible apreciar que porcentajes que pueden parecer ínfimos, dejan de serlo si los colocamos en la escala de la cantidad de instituciones que las reciben. 
Una forma de graficar el impacto de esta forma de financiamiento, y los efectos de su concentración en pocos centros, es apreciar que al recibir el Liceo Impulso la mitad del monto donado, su participación en el total de los recursos destinados por el Estado uruguayo a la educación se ubicaría en un 0,13\%. Si tenemos en cuenta que según datos de la ANEP, el organismo cuenta con $3445^{10}$ centros educativos en el país, la diferencia a favor del Liceo Impulso en la distribución del financiamiento educativo resulta notoria. Similar comparación podría realizarse con respecto a la porción de financiamiento educativo que reciben los liceos Jubilar y Providencia. De hecho existen estudios que han cuantificado los montos que estas instituciones manejan por alumno, comparándolos con los que maneja la educación pública. Un estudio realizado por Dufrechou et al. (2019) muestra como en el año 2017 por cada peso que el Estado uruguayo aportó por estudiante de la educación pública, el Liceo Jubilar dispuso de 2,76. En tanto, el mismo estudio estableció que por cada peso aportado por el Estado uruguayo, el Liceo Impulso dispuso de $\$ 2,63$ en 2015; \$2,2 en 2016; y \$ 2 en 2017 (DUFRECHOU et al., 2019).

Por otra parte, resulta significativo colocar la atención sobre aquellos actores que en definitiva son quienes definen acerca del destino de los fondos donados: las empresas donantes. Estas son las que en la práctica has alimentado con recursos una modalidad de financiamiento que, como hemos visto, se ha ido progresiva y firmemente dirigiendo hacia el financiamiento de centros educativos privados en detrimento de los públicos.

Resulta muy importante hacer notar como estos donantes han tenido la fuerza para definir el direccionamiento de recursos que en un 81,25 \% son aportados por el Estado uruguayo.

Como hemos mostrado en el apartado precedente se trata de un conjunto de empresas básicamente de corte global ligadas directamente al capital trasnacional. Estas empresas, en definitiva, deciden el destino de una porción reducida pero creciente del financiamiento educativo en Uruguay. La continuidad de la línea de investigación en la que se inserta el presente artículo deberá profundizar en la caracterización de estos grandes donantes y en el establecimiento de redes que den cuenta de sus interrelaciones tanto a nivel corporativo como de actores concretos. También es necesario dar cuenta de sus relaciones con otros actores en la esferas políticas, económicas, académicas y sociales.

Resulta interesante hacer notar como esta modalidad ha generado controversias en la sociedad uruguaya. Los sindicatos de trabajadores de la educación han sido críticos con el involucramiento de empresas privadas en la definición de recursos vinculados al financiamiento educativo. Otros actores, desde posiciones ubicadas en la derecha política del país, han defendido esta modalidad. En una nota publicada por el diario montevideano El País el 3 de julio de 2018 se recogen expresiones de Pablo da Silveira, quien como ya hemos mencionado precedentemente en aquel entonces era asesor educativo del Partido Nacional y hoy se desempeña como Ministro de Educación del gobierno de derechas instalado en Uruguay. En la referida nota de prensa se establecía:

Para Pablo Da Silveira, asesor en educación de Luis Lacalle Pou, los datos confirman algo muy distinto: 'los donantes entienden que poner plata en esas instituciones (privadas) es hacer un buen uso del dinero'. Es que el egreso en estos liceos privados

10 Datos tomados del Observatorio de la Educación de ANEP: <http://observatorio.anep.edu.uy/images/documentos/Rec_Infra_Indi1.xlsx>. 
es 'mucho mayor' que en los públicos y, por tanto, 'una inversión más efectiva'. El asesor entiende que 'la verdadera razón por la cual los sindicatos se oponen es porque hay un punto de comparación que los deja mal parados'.

Da Silveira defiende el derecho de las empresas a decidir a dónde irán a parar sus impuestos, algo 'que ya existe en varios países de Europa y Estados Unidos'. Y agregó: 'cualquier forma de escándalo ante esta financiación solo refleja ignorancia respecto a cómo funciona el mundo'.

Resulta muy interesante apreciar como el actual ministro justifica las donaciones a las instituciones privadas basándose en un criterio de "buen uso del dinero". Este uso adecuado tendría que ver con los niveles de egreso que se obtienen en estas instituciones. Lejos parece estar de la consideración de Da Silveira la reflexión acerca de la eventualidad que esos buenos resultados estén directamente vinculados a la disposición de mayores recursos que aquellos que están disponibles en la educación pública. Por otra parte, se plantea como un derecho de las empresas el decidir hacia dónde deben dirigirse sus impuestos. En este caso se trata, ni más ni menos, que apelar a que grandes transnacionales definan los destinos hacia los cuales el Estado uruguayo debe dirigir el financiamiento de la educación. Además de problemas en términos de soberanía nacional, esta perspectiva tiende a promover procesos de desfinanciamiento de la educación pública, ya que queda meridianamente claro que las empresas que hemos indagado tienen fuertes preferencias por alimentar un sistema educativo de corte privado.

De este modo, un actor clave del nuevo gobierno, se pronunciaba a favor de procesos de privatización educativa. Su justificación va en el sentido de la mayor eficiencia de la educación privada, argumento que ha sido eje de elaboraciones similares en diversos países de América Latina, como han mostrado investigaciones recientes (PERONI, 2013; BORDOLI; CONDE 2016; FELDFEBER et al., 2018) y también análisis realizados en términos más generales por autores como Harvey (2008) y Ball (2014).

\section{Consideraciones finales}

Como conclusiones iniciales del trabajo de investigación en curso, podemos mencionar algunos hallazgos que deberán ser base para la continuidad de nuestras indagaciones.

Reafirmamos la importancia de analizar los procesos de financiamiento educativo involucrados en la modalidad de Donaciones Especiales. Si bien los montos que se manejan en esta modalidad pueden parecer insignificantes en comparación con el conjunto de recursos que se movilizan en la educación uruguaya, los efectos políticos que generan no son menores.

A su vez, el peso marginal de estos montos de financiamiento cobra otra significación al constatarse que se concentran en unas pocas instituciones educativas "públicas de gestión privada". De hecho, en el caso de las tres instituciones sobre las que hemos concentrado nuestro análisis, el acceso a estos fondos les permite manejar recursos que duplican largamente los que tiene a disposición una institución educativa pública.

A partir de la asignación de los montos integrados en las donaciones realizadas en el período 2010 - 2019, las instituciones donatarias han logrado consolidar un modelo educativo en contextos de pobreza urbana que es presentado como alternativo a la educación pública. Desde aquí se sustentan perspectivas con fuerte carga ideológica que sostienen la necesidad 
de promover las modalidades de educación privada con financiamiento público a los efectos de mejorar la calidad de la educación.

Como puede apreciarse en el caso del Liceo Impulso, el análisis de estos procesos de financiamiento educativo nos muestra la existencia de fuertes convergencias entre actores del campo político, académico y empresarial a los efectos de promover procesos de privatización educativa.

En lo que hace a los actores del ámbito empresarial, resulta muy significativo subrayar el peso de empresas vinculadas al capital trasnacional en el aporte de fondos por la modalidad de Donaciones Especiales. De hecho estas empresas han sido las protagonistas de un proceso de concentración de donaciones en un reducido conjunto de instituciones privadas. Estas donaciones han permitido a las instituciones mencionadas ser la punta de lanza de una estrategia argumentativa que busca sustentar la necesidad de avanzar en procesos de privatización educativa en Uruguay. Un elemento que no debemos dejar de tener presente en ningún momento es que las empresas donantes recuperan hasta el $81,25 \%$ de los montos donados a través de exoneraciones fiscales. Esta renuncia fiscal que realiza el Estado uruguayo hace que el mismo renuncie a establecer el destino de una parte de los rubros que destina al financiamiento educativo, quedando esta decisión en manos de un conjunto de empresas privadas con fuerte interés en generar procesos de privatización educativa en el país.

La magnitud de los procesos de conjunción de actores políticos, académicos y empresariales que confluyen para el direccionamiento de fondos públicos hacia instituciones privadas, así como para la promoción de procesos de privatización educativa en Uruguay, torna sumamente necesaria la continuidad de trabajos de investigación que profundicen en esta temática.

\section{Referencias}

BALL, S. Política social y educativa, empresa social, hibridación y nuevas comunidades discursivas. Propuesta Educativa, n. 36, año 20, v. 2; p. 25-34, 2011.

BALL, S. Globalización, mercantilización y privatización: tendencias internacionales en Educación y Política Educativa. Archivos Analíticos de Políticas Educativas, v. 22, n. 41, 2014. Disponibles en: <http://dx.doi.org/10.14507/epaa.v22n41.2014>. Aceso en: 02 mar. 2020.

BALL, S. J.; YOUDELL, D. Hidden privatisation in public education. Brussels: Education International, 2008.

BELLEI, C.; ORELLANA, V. What Does "Education Privatisation" Mean? Conceptual Discussion and Empirical Review of Latin American Cases. (ESP Working Paper Series - Open Society Foundations n. 62). New York: Open Society Foundations, 2014.

BORDOLI, E.; CONDE, S. El progresivo encanto por la gestión privada: análisis de los modelos de la gestión pública-privada en enseñanza media en Uruguay (2002-2013). Educação \& Sociedade, Campinas, v. 37, n. 134, p. 73-90, 2016. 
Disputas sobre el sentido de la educación pública

BORDOLI, E.; CONDE, S. El proyecto educativo conservador en Uruguay en los albores del siglo XXI: avance privatizador y tutela ministerial. Práxis Educativa, Ponta Grossa, v. 15, p. $1-21,2020$.

BORDOLI, E. et al. Privatización educativa en Uruguay: políticas, actores y posiciones. Montevideo: Internacional de la Educación, 2017.

CAETANO, M. O protagonismo do setor privado na reforma do Ensino Médio no Brasil: o Instituto Unibanco e suas relações. En: PERONI, V.; DE LIMA, P.; KADER, C. (Org.). Redefinições das fronteiras entre o público e o privado. Implicações para a democratização da educação. São Leopoldo: Oikos, 2018.

CIPPEC. Aportes financieros estatales a la educación privada. Programa de Monitoreo de la Ley de Financiamiento Educativo. Buenos Aires, 2007.

DA SILVEIRA, P. La segunda reforma. Montevideo: Fundación Bank Boston, 1995.

DOBERTI, J. El subsidio a la educación privada en la Argentina y Chile: un estudio comparativo. En: ESTEVEZ, A. (Comp.). Reforma managerialista del Estado. La nueva gerencia pública, calidad total y tecnocracia. Buenos Aires: Cooperativas, 2001.

DONACIONES a liceos privados son 18 veces mayores que a ANEP. EI País, Montevideo, 3 jul. 2018. Disponible en: <https://www.elpais.com.uy/informacion/educacion/donacionesliceos-privados-son->veces-mayores-anep.html>. Acceso: 5 jul. 2020.

DUFRECHOU, $\mathrm{H}$. et al. El avance privatizador en la educación uruguaya: discursos y políticas. Montevideo: Internacional de la Educación, 2019.

FELDFEBER, M., et al. La privatización educativa en Argentina. Ciudad Autónoma de Buenos Aires: CTERA, 2020.

HARVEY, D. O neoliberalismo: história e implicações. São Paulo: Loyola, 2008.

INEED. Instituto Nacional de Evaluación Educativa. Informe sobre el estado de la educación en Uruguay 2015-2016. Montevideo: INEED, 2017.

LEHER, R. Estrategias de mercantilización de la educación y tiempos desiguales de los tratados de libre comercio: el caso de Brasil. En: GENTILI, P.; FROGOTTO, G.; LEHER, R.; STUBRIN, F. (Comp.). Políticas de privatización, espacio público y educación en América Latina. Rosario: Homo Sapiens, 2009.

MANCEBO, M. Fronteras porosas entre la educación pública y la privada en Uruguay. En: PERONI, V.; VALIM, P.; KADER, C. (Org.). Redefiniciones das fronteiras entre o público e o privado: implicações para a democratização da educação. Porto Alegre: Editora OIKOS, 2018. p. 30-46.

MARTINIS, P. Modelos educativos privatizadores y disputas por el derecho a la educación. En: SERPAJ. Derechos humanos en el Uruguay. Informe 2019. Montevideo: Tradinco, 2019.

MOSCHETTI, M. et al. The increasing role of non-State actors in education policy-making. Evidence from Uruguay. Journal of Education Policy, v. 34, 2019. Disponible en: <https://doi.org/10.1080/02680939.2018.1562569>. Acceso: 5 jul. 2020. 
Disputas sobre el sentido de la educación pública

PERONI, V.; DE LIMA, P.; KADER, C. (2018) (Org.). Redefinições das fronteiras entre o público e o privado: implicações para a democratização da educação. São Leopoldo: Oikos, 2018.

PERONI, V. Redefinições das fronteiras entre o público e o privado: implicações para a democratização da educação. Brasilia: Liber Livro, 2013.

VERGER, A.; FONTDEVILA, C.; ZANCAJO, A. The privatisation of education: a political economy of global education reform. New York: Teachers College Press, 2016.

VERGER, A.; MOSCHETTI, M.; FONTDEVILA, C. La privatización educativa en América Latina: Una cartografía de políticas, tendencias y trayectorias. Bruselas: Internacional de la Educación, 2017.

URUGUAY. Ley 16226. Diario Oficial, Montevideo, 1991. Disponible en: $<$ https://parlamento.gub.uy>. Acceso: 5 jul. 2020.

URUGUAY (2007). Ley 18083. Diario Oficial, Montevideo, 2007. Disponible en: $<$ https://parlamento.gub.uy>. Acceso: 5 jul. 2020.

URUGUAY (2012). Ley 18834. Diario Oficial, Montevideo, 2012. Disponible en: $<$ https://parlamento.gub.uy>. Acceso: 5 jul. 2020.

URUGUAY (2014). Ley 19149. Diario Oficial, Montevideo, 2014. Disponible en: <https://parlamento.gub.uy>. Acceso: 5 jul. 2020.

URUGUAY (2018). Ley 19438. Diario Oficial, Montevideo, 2018a. Disponible en: <https://parlamento.gub.uy>. Acceso: 5 jul. 2020.

URUGUAY (2018). Ley 19670. Diario Oficial, Montevideo, 2018b. Disponible en: <https://parlamento.gub.uy>. Acceso: 5 jul. 2020.

URUGUAY. Ministerio de Educación y Cultura. Logro y nivel educativo de la población. Montevideo: MEC, 2018c.

URUGUAY. Ministerio de Educación y Cultura. Anuario estadístico 2018. Montevideo: MEC, 2019a.

URUGUAY. Ministerio de Economía y Finanzas Rendición de cuentas y balance de ejecución presupuestal 2018. Montevideo: MEF, 2019b.

URUGUAY. Ministerio de Economía y Finanzas. Rendición de cuentas y balance de ejecución presupuestal 2019. Montevideo: MEF, 2020.

Pablo Martinis es Profesor Titular del Instituto de Educación de la Facultad de Humanidades y Ciencias de la Educación (Universidad de la República, Uruguay).

ORCID: https://orcid.org/0000-0002-1210-0098

E-mail: pablomartinis@gmail.com

Recibido en 29 de julio de 2020 Aprobado en 29 de agosto de 2020 


\section{Editores do volume 10}

Márcia Aparecida Jacomini - Universidade Federal de São Paulo, Brasil

José Marcelino de Rezende Pinto - Universidade de São Paulo, Brasil

\section{Comitê Editorial}

Nalú Farenzena - Universidade Federal do Rio Grande do Sul, Brasil

Juca Gil - Universidade Federal do Rio Grande do Sul, Brasil

Theresa Adrião - Universidade Estadual de Campinas, Brasil

Ângelo Ricardo de Souza - Universidade Federal do Paraná, Brasil

\section{Conselho Editorial}

\section{Alejandro Morduchowicz}

Universidad Pedagógica, Provincia de Buenos Aires, Argentina

Andréa Barbosa Gouveia

Universidade Federal do Paraná, Brasil

Fernanda Saforcada

Universidade de Buenos Aires, Argentina

Jacques Velloso

Universidade de Brasília, Brasil

João Monlevade

Senado Federal, Brasil

Jorge Abrahão de Castro

Instituto de Pesquisa Econômica Aplicada / IPEA, Brasil

Lisete Regina Gomes Arelaro

Universidade de São Paulo, Brasil

Luis Carlos Sales

Universidade Federal do Piauí, Brasil

Luiz de Sousa Junior

Universidade Federal da Paraíba, Brasil

Luiz Fernandes Dourado

Universidade Federal de Goiás, Brasil

Magna França

Universidade Federal do Rio Grande do Norte, Brasil

Marcos Edgar Bassi

Universidade Federal de Santa Catarina, Brasil

Maria Angélica Pedra Minhoto

Universidade Federal de São Paulo, Brasil

Maria Beatriz Luce

Universidade Federal do Rio Grande do Sul, Brasil

Maria Dilnéia Espíndola Fernandes

Universidade Federal de Mato Grosso do Sul, Brasil

Nelson Cardoso do Amaral

Universidade Federal de Goiás, Brasil

Nicholas Davies

Universidade Federal Fluminense, Brasil

Robert E. Verhine

Universidade Federal da Bahia, Brasil

Romualdo Portela de Oliveira

Universidade de São Paulo, Brasil

Rosana Gemaque Rolim

Universidade Federal do Pará, Brasil

Rubens Barbosa de Camargo

Universidade de São Paulo, Brasil

Theresa Adrião

Universidade Estadual de Campinas, Brasil

Tristan McCowan

University of London, Reino Unido

Vera Jacob

Universidade Federal do Pará, Brasil

Vera Peroni

Universidade Federal do Rio Grande do Sul, Brasil

Vitor Henrique Paro

Universidade de São Paulo, Brasil

\section{Equipe editorial}

Apoio ao Comitê Editorial: Caio Cabral da Silva

Diagramação, Revisão de português e normalização: Edson Leonel de Oliveira

Revisão de inglês: Sabrina Ferreira

Fineduca - Revista de Financiamento da Educação

Associação Nacional de Pesquisa em

Financiamento da Educação

e-mail: revista.fineduca@gmail.com | site: http://seer.ufrgs.br/fineduca 